\title{
Erratum: Prevalence and Awareness of Stroke and Other Comorbidities Associated with Diabetes in Northwest India
}

Vivek Podder ${ }^{1, *}$ Vinod Srivastava ${ }^{2, *}$ Saurabh Kumar ${ }^{3, *} \quad$ Raghuram Nagarathna $^{4}$ Madhava Sai Sivapuram ${ }^{5}$ Navneet Kaur ${ }^{3,6} \quad$ Kanupriya Sharma ${ }^{3}$ Amit Kumar Singh ${ }^{4}$ Neeru Malik ${ }^{7}$ Akshay Anand ${ }^{3, * *}$ Hongasandra R. Nagendra ${ }^{4}$

${ }^{1}$ Department of General Medicine, Kamineni Institute of Medical Sciences, Narketpally, Telangana, India

2Department of Social Work, University of Kentucky, Lexington, Kentucky, United States

${ }^{3}$ Department of Neurology, Neuroscience Research Lab, Postgraduate Institute of Medical Education and Research (PGIMER), Chandigarh, India

${ }^{4}$ Swami Vivekananda Yoga Anusandhana Samsthana, Bengaluru, India

5 Department of General Medicine, Dr. Pinnamaneni Siddhartha Institute of Medical Sciences and Research Foundation, Chinna-Avutapalli, Krishna, Andhra Pradesh, India

${ }^{6}$ Department of Physical Education, Panjab University, Chandigarh, India

${ }^{7}$ Department of Yoga, Dev Samaj College of Education, Panjab University, Chandigarh, India

J Neurosci Rural Pract 2020;11(03): e1
Address for correspondence Raghuram Nagarathna, Medical Director, Arogya Dham, SVYASA, Bengaluru 560105, India (e-mail: rnagaratna@gmail.com);

Akshay Anand, PhD, Department of Neurology, PGIMER, Chandigarh, 160014, India (e-mail: akshay1anand@rediffmail.com).

\section{Erratum}

\footnotetext{
${ }^{*}$ Equal first authors

** Co-corresponding author
}

DOI https://doi.org/

10.1055/s-0040-1719205

ISSN 0976-3147.
It has been brought to the publisher's attention that the Address for Correspondence was incorrect in the above article in Journal of Neurosciences in Rural Practice, published in the issue 11(03) (DOI: 10.1055/s-0040-1709369). The correct details appear as above.
(C)2020 Association for Helping Neurosurgical Sick People
License terms

()(1) $\odot \circledast$ 